\title{
Venous Thromboembolism in Systemic Sclerosis: Prevalence, Risk Factors, and Effect on Survival
}

\author{
Sindhu R. Johnson, Nabil Hakami, Zareen Ahmad, and Duminda N. Wijeysundera
}

ABSTRACT. Objective. Whether systemic sclerosis (SSc) confers increased risk of venous thromboembolism (VTE) is uncertain. We evaluated the prevalence, risk factors, and effect of VTE on SSc survival. Methods. A cohort study was conducted of subjects with SSc who fulfilled the American College of Rheumatology/European League Against Rheumatism classification criteria between 1970 and 2017. Deep vein thrombosis was defined as thrombus on extremity ultrasound. Pulmonary embolism was defined as thrombus on thorax computed tomography angiogram. Risk factors for VTE and time to all-cause mortality were evaluated.

Results. Of the 1181 subjects, 40 (3.4\%) experienced VTE events. The cumulative incidence of VTE was 2.7 (95\% CI 1.9-3.7) per 1000 patient-years. Pulmonary arterial hypertension (PAH; OR 3.77, 95\% CI 1.83-8.17), peripheral arterial disease (OR 5.31, 95\% CI 1.99-12.92), Scl-70 (OR 2.45, 95\% CI 1.07-5.30), and anticardiolipin antibodies (OR 5.70,95\% CI 1.16-21.17) were predictors of VTE. There were 440 deaths. There was no difference in survival between those with and without VTE (HR 1.16, 95\% CI 0.70-1.91). Interstitial lung disease (HR 1.54, 95\% CI 1.27-1.88) and PAH (HR $1.35,95 \%$ CI $1.10-1.65)$ were predictors of mortality.

Conclusion. The risk of VTE in SSc is comparable to the general population. The presence of PAH, peripheral arterial disease, Scl-70, and anticardiolipin antibodies are risk factors for VTE. VTE does not independently predict SSc survival. (First Release April 15 2018; J Rheumatol 2018;45:942-6; doi:10.3899/jrheum.170268)

Key Indexing Terms: SYSTEMIC SCLEROSIS PULMONARY EMBOLISM
SCLERODERMA

\section{VENOUS THROMBOEMBOLISM DEEP VEIN THROMBOSIS}

From the Toronto Scleroderma Program, Mount Sinai Hospital, Toronto Western Hospital; Division of Rheumatology, Department of Medicine, University of Toronto; Institute of Health Policy, Management and Evaluation; Department of Anesthesia and Institute of Health Policy, Management and Evaluation, University of Toronto; Department of Anesthesia and Pain Management, Toronto General Hospital, Toronto, Ontario, Canada; King Fahad Central Hospital, Ministry of Health, Jizan, Saudi Arabia.

Dr. Johnson is supported by a Canadian Institutes of Health Research Clinician Scientist Award, the Oscar and Eleanor Markovitz. Fund for Scleroderma Research, and the Freda Fejer Fund of the Arthritis Research Foundation. Dr. Wijeysundera is supported in part by a New Investigator Award from the Canadian Institutes of Health Research and a Merit Award from the Department of Anesthesia at the University of Toronto.

S.R. Johnson, MD, PhD, Toronto Scleroderma Program, Mount Sinai Hospital, Toronto Western Hospital, Division of Rheumatology, Department of Medicine, University of Toronto, and Institute of Health Policy, Management and Evaluation, University of Toronto; N. Hakami, MD, Toronto Scleroderma Program, Mount Sinai Hospital, Toronto Western Hospital, Division of Rheumatology, Department of Medicine, University of Toronto, and King Fahad Central Hospital, Ministry of Health; Z. Ahmad, MD, Toronto Scleroderma Program, Mount Sinai Hospital, Division of Rheumatology, Department of Medicine, University of Toronto; D.N. Wijeysundera, MD, PhD, Department of Anesthesia and Pain Management, Toronto General Hospital, and Department of Anesthesia and Institute of Health Policy, Management and Evaluation, University of Toronto.

Address correspondence to Dr. S. Johnson, Division of Rheumatology, Ground Floor, East Wing, Toronto Western Hospital, 399 Bathurst St., Toronto, Ontario M5T 2S8, Canada.E-mail: Sindhu.Johnson@uhn.ca Accepted for publication December 14, 2017.
Venous thromboembolism (VTE) is a vascular phenomenon that includes deep vein thrombosis (DVT) and pulmonary embolism (PE). It is recognized that active systemic inflammation can augment coagulability and lead to thrombosis ${ }^{1}$. Systemic sclerosis ( $\mathrm{SSc}$ ) is characterized by vascular lesions, inflammation, and fibrosis ${ }^{2}$. Vasculopathy is associated with reperfusion injury and endothelial dysfunction, which may influence the onset of local thrombotic complications $s^{3,4,5}$. Whether there is an increased risk of VTE in SSc is being debated.

A metaanalysis evaluating the risk of VTE in inflammatory rheumatic disease concluded that inflammatory rheumatologic diseases were associated with a 3 -fold higher risk of VTE compared to the general population ${ }^{1}$. However, the SSc-specific data were attributable to only 4 studies. One cohort study reported that people with immune-mediated disease may be at increased risk of subsequent VTE. However, that study included a range of immune-mediated diseases and was limited to inpatients or daytime specialist care $^{6}$. Another retrospective cohort study of individuals admitted to hospital because of an autoimmune disorder found that autoimmune disorders were associated with increased risk of PE during the first year after admission. The standardized incidence ratio for SSc was 1.61 (95\% CI

Personal non-commercial use only. The Journal of Rheumatology Copyright $\subset$ C 2018. All rights reserved. 
1.45-1.79). This study was potentially biased to the more severely ill, who required inpatient day hospital care ${ }^{7}$. The remaining 2 studies relied on administrative data. One population-based cohort study reported patients with SSc had a 10.5-fold increased risk of DVT and a 7.0-fold increased risk of PE compared with the general population ${ }^{8}$. Another population-based cohort study reported a relative risk of DVT of 5.0 (95\% CI 2.7-9.4) and relative risk of PE of 4.0 (95\% CI 2.1-7.6) $)^{9}$. However, both studies relied on International Classification of Diseases codes for case-finding and may have included other prothrombotic diseases such as systemic lupus erythematosus or overlap syndromes. Thus, it remains uncertain whether SSc confers an increased risk of VTE. Improved understanding of the relationship and effect of VTE on SSc may have implications for screening and anticoagulation treatment.

The aim of our study was to evaluate the epidemiology of VTE in SSc. We evaluated the cumulative incidence of VTE and compared our findings to that of the general population to ascertain whether SSc is associated with an increased risk of VTE. We evaluated risk factors for the development of VTE in SSc, and the effect of VTE on SSc survival.

\section{MATERIALS AND METHODS}

Subjects. The Toronto Scleroderma Program, a health network comprising 3 academic hospitals, is the largest single center longitudinal cohort in Canada ${ }^{10}$. Subjects who fulfilled the American College of Rheumatology/ European League Against Rheumatism classification criteria for SSc were included ${ }^{11}$. Subjects with localized scleroderma, overlap syndromes, undifferentiated connective tissue disease, and an inherited thrombophilia were excluded. The study period was 1970-2017.

Outcomes. DVT was defined as the presence of thrombus of the upper extremity, femoral, or popliteal veins, or distal (calf) thrombosis with proximal extension on Doppler ultrasound. PE was defined as the presence of thrombus on thorax computed tomography (CT) angiogram. Ultrasound and CT scan were performed based on clinical suspicion. D-dimers and Well's score were not used to stratify risk. Comparing those with and without VTE, we evaluated differences in disease duration, subtype, SSc manifestations, and comorbidities.

Differences in baseline characteristics between those with and without VTE included male sex, subtype of SSc (limited or diffuse, ascertained at baseline but revised if limited evolved into diffuse), SSc manifestations [calcinosis, Raynaud phenomenon, digital ulceration, symptomatic esophageal dysmotility on history, telangiectasia, abnormal nailfold capillaries on visual inspection, interstitial lung disease (ILD; forced vital capacity $<70 \%$ ), and bibasilar reticular abnormalities with minimal ground glass on high resolution CT thorax $]^{10}$, pulmonary arterial hypertension [PAH; mean pulmonary artery pressure (mPAP) $>25 \mathrm{mmHg}$ and pulmonary capillary wedge pressure $(\mathrm{PCWP})<15 \mathrm{mmHg}$ by right heart catheterization $]^{12}$, renal crisis (acute renal failure, new onset hypertension, normal or mild proteinuria on urinalysis, microangiopathic hemolytic anemia), and serology (Scl-70 centromere antibodies).

Analysis. Descriptive statistics and relative risk (RR) with 95\% CI were used to summarize the data. The prevalence of VTE in our cohort was compared to the general population using the Poisson distribution. We also evaluated prevalence by age, race, and sex. Logistic regression models were fit to evaluate risk factors for VTE. We evaluated model discrimination using the c statistic, and model calibration using the Hosmer-Lemeshow statistic. Patients who were alive on January 1, 2017, were right censored. All-cause mortality was the outcome for the survival models. The survival status of subjects who were lost to followup was systematically tracked through the hospital electronic record, family physician, referring physician, and online obituary databases. This approach has been shown to be a valid and reliable method of obtaining robust mortality data ${ }^{13}$, and has been successfully implemented in other studies ${ }^{10,14,15}$. Differences in survival were evaluated using Kaplan-Meier and Cox proportional hazards models. Analyses were conducted using RStudio (version 0.97.248).

Ethics. Institutional research ethics board approval was obtained prior to the conduct of this study (UHN REB 15-9209-AE, MSH REB 15-0256-C), and the study was conducted in compliance with the Helsinki Agreement.

\section{RESULTS}

Subjects. There were 1181 subjects [971 (82\%) females, 210 $(18 \%)$ males] who fulfilled eligibility criteria. In our cohort, there were 2 subjects with inherited thrombophilia (protein $\mathrm{C}$ deficiency, protein $\mathrm{S}$ deficiency), who were excluded from our study.

Frequency. There were 40 patients $(3.4 \%)$ with unique VTE events. There were 20 DVT (1.7\%) and 26 PE (2.2\%) events. Of these, $6(0.5 \%)$ had both a DVT and PE. The prevalence of VTE was 2.7 (95\% CI 1.9-3.7) per 1000 patient years. Compared to a general population prevalence of VTE of 2 cases per 1000 persons ${ }^{16}$, the prevalence of VTE in SSc did not differ significantly from the general population $(p=0.07)$. We note that although $0.5 \%$ had both DVT and PE, subjects were not double-counted in the estimation of prevalence.

In subgroup analyses, the prevalence of VTE in SSc appears to increase with age, similar to the general population. There appears to be no differences in the incidence of VTE between males and females. The prevalence of VTE appears to be higher among African Americans compared to whites and Asians (Table 1).

Risk factors for VTE. Comparisons of SSc characteristics and comorbidities between those with and without VTE are summarized in Table 2.

In unadjusted bivariate comparisons, the presence of these conditions was more prevalent in subjects who developed VTE: ILD (RR 1.67, 95\% CI 1.26-2.20), PAH (RR 2.18,

Table 1. Estimated prevalence of VTE by ethnicity, age, and sex.

\begin{tabular}{lc}
\hline Characteristics & Prevalence per 1000 Patient-yrs \\
\hline Race/ethnicity & \\
$\quad$ White & 2.40 \\
African American & 6.94 \\
$\quad$ Asian & 0.85 \\
$\quad$ East Asian & 1.43 \\
Age, yrs & \\
17-44 & 1.54 \\
45-79 & 4.07 \\
$\geq 80$ & 28.95 \\
Sex & \\
$\quad$ Male & 3.85 \\
Female & 2.52 \\
\hline
\end{tabular}

VTE: venous thromboembolism. Personal non-commercial use only. The Journal of Rheumatology Copyright (c) 2018. All rights reserved. 
Table 2. Comparison of SSc subjects with and without VTE. Values are n (\%) unless otherwise specified.

\begin{tabular}{lccc}
\hline Characteristics & VTE, $\mathrm{n}=40$ & No VTE, $\mathrm{n}=1141$ & RR $(95 \% \mathrm{CI})$ \\
\hline Diffuse subtype & $12(30)$ & $383(34)$ & $1.04(0.84-1.30)$ \\
Male sex & $9(23)$ & $201(18)$ & $1.28(0.71-2.30)$ \\
Calcinosis & $10(25)$ & $305(27)$ & $1.00(0.99-1.00)$ \\
Raynaud phenomenon & $39(98)$ & $1087(95)$ & $1.02(0.97-1.08)$ \\
Esophageal dysmotility & $35(88)$ & $981(86)$ & $1.02(0.90-1.15)$ \\
Telangiectasia & $29(73)$ & $846(74)$ & $0.98(0.81-1.19)$ \\
ILD & $23(58)$ & $393(34)$ & $1.67(1.26-2.20)^{*}$ \\
PAH & $28(70)$ & $367(32)$ & $2.18(1.75-2.71)^{*}$ \\
Renal crisis & $5(13)$ & $74(6)$ & $1.93(0.82-4.50)$ \\
Abnormal nailfold capillaries & $14(35)$ & $354(31)$ & $1.12(0.73-1.74)$ \\
Digital ulcers & $13(33)$ & $386(34)$ & $0.96(0.61-1.51)$ \\
Scl-70 antibody & $11(28)$ & $186(16)$ & $1.69(1.00-2.84)^{*}$ \\
ACA & $5(13)$ & $215(19)$ & $0.66(0.29-1.52)$ \\
Lupus anticoagulant & $(0)$ & $7(0.6)$ & NA \\
aCL & $3(8)$ & $14(1)$ & $6.11(1.83-20.4)^{*}$ \\
Coronary artery disease & $9(23)$ & $98(9)$ & $2.62(1.43-4.80)^{*}$ \\
Hypertension & $11(28)$ & $237(21)$ & $1.32(0.79-2.22)$ \\
Diabetes mellitus & $6(15)$ & $61(5)$ & $2.81(1.29-6.10)^{*}$ \\
Hyperlipidemia & $5(13)$ & $78(7)$ & $1.83(0.78-4.27)$ \\
Peripheral arterial disease & $8(20)$ & $41(4)$ & $5.57(2.79-11.08)^{*}$ \\
Cancer & $7(18)$ & $127(11)$ & $1.57(0.79-3.14)$ \\
Stroke & $2(5)$ & $30(3)$ & $1.90(0.47-7.68)$ \\
Atrial fibrillation & $6(15)$ & $45(4)$ & $3.80(1.72-8.39)^{*}$ \\
\hline
\end{tabular}

* Denotes statistical significance. VTE: venous thromboembolism; RR: relative risk; ILD: interstitial lung disease; PAH: pulmonary arterial hypertension; ACA: anticentromere antibodies; NA: not available; aCL: anticardiolipin antibodies.

1.75-2.71), Scl-70 antibody (RR 1.69, 95\% CI 1.00-2.84), anticardiolipin antibodies (aCL; RR 6.11,95\% CI 1.83-20.4), coronary artery disease (RR 2.62, 95\% CI 1.43-4.80), diabetes mellitus (RR 2.81, 95\% CI 1.29-6.10), and peripheral arterial disease (RR 5.57, 95\% CI 2.79-11.08). The subjects who had the presence of a lupus anticoagulant or aCL did not have antiphospholipid antibody syndrome. Independent predictors of VTE in SSc were PAH (OR 3.77, 95\% CI 1.83-8.17), peripheral arterial disease (OR 5.31, 95\% CI 1.99-12.92), Scl-70 (OR 2.45, 95\% CI 1.07-5.30), and aCL (OR 5.70, 95\% CI 1.16-21.17; Table 3). Subjects with ILD more frequently experienced DVT (RR $2.85,95 \%$ CI 1.08-7.54) but not PE (RR 1.82, 95\% CI 0.89-3.70). There

Table 3. Summary of logistic regression model evaluating baseline predictors of survival.

\begin{tabular}{lccc}
\hline Variables & OR & $95 \%$ CI & $\mathrm{p}$ \\
\hline PAH & 3.77 & $1.83-8.17$ & $<0.001$ \\
Scl-70 antibody & 2.45 & $1.07-5.30$ & 0.03 \\
aCL & 5.70 & $1.16-21.17$ & 0.02 \\
Peripheral arterial disease & 5.31 & $1.99-12.92$ & $<0.001$ \\
Diabetes mellitus & 2.32 & $0.79-5.85$ & 0.03 \\
ILD & 1.6 & $0.80-3.35$ & 0.18 \\
Coronary artery disease & 1.94 & $0.77-4.47$ & 0.14 \\
Atrial fibrillation & 2.26 & $0.73-6.00$ & 0.12 \\
\hline
\end{tabular}

PAH: pulmonary arterial hypertension; ILD: interstitial lung disease; aCL: anticardiolipin antibodies. was no significant difference in the occurrence of DVT (RR $0.95,95 \%$ CI $0.46-1.97$ ) or PE (RR $0.98,95 \%$ CI $0.74-1.30$ ) between SSc subtypes.

Survival. There were 440 deaths. The probability of short-term (1-, 2-, 3-, 4-, 5-yr) and longterm (10-, 15-, 20-yr) survival, and median survival times between SSc subjects with and without VTE, are summarized in Table 4.

There were no significant differences in short-term survival between groups (log-rank test, $\mathrm{p}=0.54)$. The Kaplan-Meier curves in Figure 1 suggest worsening longterm survival for the VTE group. There was no significant difference in the unadjusted survival between the 2 groups (HR 1.16, 95\% CI 0.70-1.91). This was further attenuated

Table 4. Survival probabilities and median survival time. Values are \% (95\% CI), except where indicated.

\begin{tabular}{lcc}
\hline Probability of Survival, yrs & VTE & No VTE \\
\hline 1 & $100(100-100)$ & $97.7(96.9-98.6)$ \\
2 & $97.4(92.4-100)$ & $95.4(94.1-96.6)$ \\
3 & $94.6(87.6-100)$ & $93.7(92.3-95.2)$ \\
4 & $89.0(79.4-99.8)$ & $90.9(89.1-92.6)$ \\
5 & $80.4(68.4-94.6)$ & $88.7(86.7-90.6)$ \\
10 & $67.0(52.6-85.4)$ & $75.8(73.1-78.7)$ \\
15 & $59.1(43.8-79.7)$ & $66.3(63.1-69.8)$ \\
20 & $42.3(26.0-68.7)$ & $54.3(50.4-58.5)$ \\
Median survival time, yrs & 15.6 & 22.0 \\
\hline
\end{tabular}

VTE: venous thromboembolism. 


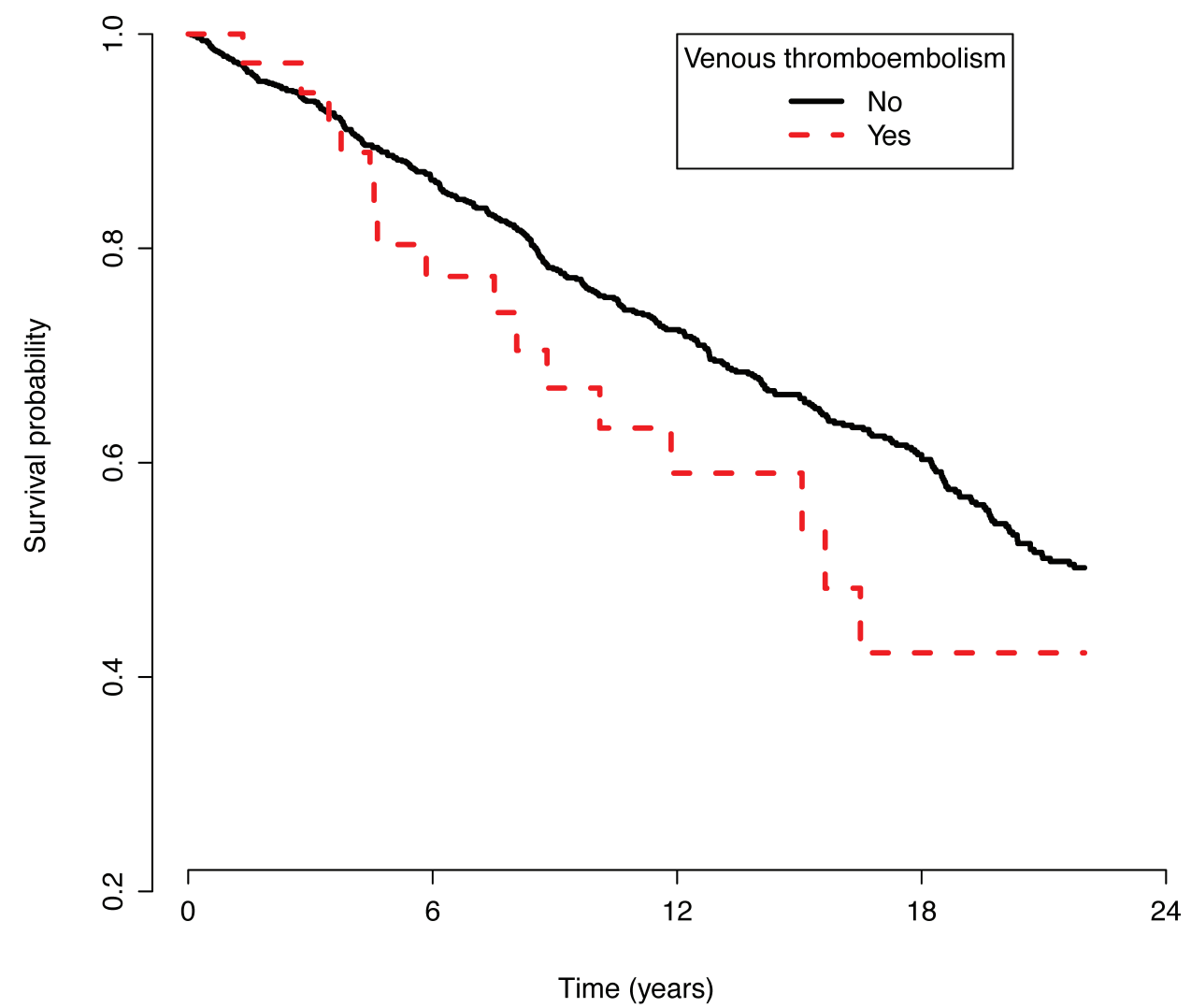

Figure 1. Kaplan-Meier survival curves of subjects with systemic sclerosis, with and without venous thromboembolism (log-rank test, $\mathrm{p}=0.54)$.

(HR 1.02, 95\% CI 0.62-1.67) after adjustment for the presence of ILD, PAH, Scl-70 antibody, aCL, coronary artery disease, diabetes mellitus, and peripheral arterial disease. Only the presence of ILD (HR 1.54, 95\% CI 1.27-1.88) or PAH (HR 1.35, 95\% CI 1.10-1.65) were independent predictors of mortality.

\section{DISCUSSION}

Vascular abnormalities in SSc including microvascular changes, endothelial dysfunction, impaired angiogenesis, thrombotic arteriopathy, platelet activation, and increased aggregability provide a plausible biologic rationale for the development of VTE in $\mathrm{SSc}^{5,17}$. It has been suggested that $\mathrm{SSc}$ is an underrecognized risk factor for VTE, but the evidence to support this is limited ${ }^{1}$. The cumulative incidence of VTE in SSc of 2.7 per 1000 patient-years in our study is comparable to the VTE cumulative incidence of $3.13 \%(95 \%$ CI $1.73-4.52 \%$ ) reported in a 2014 metaanalysis ${ }^{1}$. However, we found the cumulative incidence is not significantly higher than observed in the general population ${ }^{16}$. This suggests that unlike other rheumatologic disease such as rheumatoid arthritis and systemic lupus erythematosus, SSc is not an independent risk factor for VTE.

Classic risk factors of VTE including immobility, surgery, obesity, and malignancy have been well documented ${ }^{16}$. In
SSc, it has been hypothesized that the diffuse cutaneous subtype may be more predisposed to VTE, because diffuse $\mathrm{SSc}$ is associated with elevated plasma D-dimer levels, conferring a possible prothrombotic state ${ }^{3}$. We found no significant differences in the incidence of DVT or PE between the subtypes, suggesting both subtypes are at equal risk of VTE. We found that the presence of PAH, peripheral arterial disease, Scl-70, and aCL were independent risk factors for VTE in SSc. That is, these risk factors (and the other baseline characteristics we evaluated) occurred prior to the occurrence of VTE. This may have implications in the evaluation of patients with SSc-PAH presenting with worsening dyspnea, because our data suggest that PAH is a risk factor for VTE in SSc.

We found that SSc-ILD subjects more frequently experienced DVT. This is consistent with the observation that individuals with idiopathic pulmonary fibrosis have higher incidence rates of $\mathrm{DVT}^{18}$. The development of DVT in subjects with SSc-ILD may be attributable to immobility. Although infrequent, aCL can occur in the setting of SSc and are associated with thrombosis ${ }^{19}$. Together, these observations suggest that SSc does not independently confer a venous hypercoagulable state. Rather, when VTE occurs in SSc, one should consider other attributable causes.

Finally, we found no difference in short-term survival

Personal non-commercial use only. The Journal of Rheumatology Copyright $\subset$ 2018. All rights reserved. 
between SSc subjects with and without VTE. This may address the fact that there is treatment for VTE and it is effective. However, the survival curves suggested worsening longterm survival for SSc subjects with VTE. ILD and PAH remained independent predictors of mortality.

To our knowledge, this is the first study with primary data collection to evaluate the epidemiology of VTE in SSc in the outpatient setting using a large, well-characterized SSc cohort. We evaluated the effect of VTE on survival using robust mortality data with a long followup duration ${ }^{10,13}$. A potential limitation is that VTE may have occurred in subjects that were lost to followup and not detected in our study. This may result in our underestimation of the prevalence. Another potential limitation to our work is that we report only symptomatic VTE, because investigations for VTE were based on symptoms and/or signs. We did not systematically screen all asymptomatic subjects or ascertain risk prior to investigation. Although this may result in our underestimation of the rate of VTE in SSc, this reflects real-world practice. The population-based estimates of VTE, with which we compare our findings, used the same screening approach ${ }^{16}$.

The discordance between our findings and the previously published works may have occurred for a few reasons. It is worth noting that the other Canadian administrative data study involved a distinct population from ours (province of British Columbia vs Ontario). None of the subjects with VTE in our study were taking oral contraceptive or hormone replacement therapy, nor did they have a previous history of DVT or surgery prior to the VTE event. We were not able to adjust for immobility, but this may be colinear with advanced ILD or PAH. In our study, there was no significant difference in the occurrence of cancer between those with and without VTE events.

VTE is an infrequent but important occurrence in SSc. Improving our understanding about the burden and causes of VTE in SSc has the potential to prevent and reduce morbidity and mortality from $\mathrm{VTE}^{16}$.

\section{REFERENCES}

1. Lee JJ, Pope JE. A meta-analysis of the risk of venous thromboembolism in inflammatory rheumatic diseases. Arthritis Res Ther 2014;16:435.

2. Johnson SR, Granton JT. Pulmonary hypertension in systemic sclerosis and systemic lupus erythematosus. Eur Respir Rev 2011;20:277-86.

3. Lippi G, Volpe A, Caramaschi P, Salvagno GL, Montagnana M, Guidi GC. Plasma D-dimer concentration in patients with systemic sclerosis. Thromb J 2006;4:2.
4. Matucci-Cerinic M, Kahaleh B, Wigley FM. Review: evidence that systemic sclerosis is a vascular disease. Arthritis Rheum 2013;65:1953-62.

5. Johnson SR, Granton JT, Mehta S. Thrombotic arteriopathy and anticoagulation in pulmonary hypertension. Chest 2006;130:545-52.

6. Ramagopalan SV, Wotton CJ, Handel AE, Yeates D, Goldacre MJ. Risk of venous thromboembolism in people admitted to hospital with selected immune-mediated diseases: record-linkage study. BMC Med 2011;9:1

7. Zoller B, Li X, Sundquist J, Sundquist K. Risk of pulmonary embolism in patients with autoimmune disorders: a nationwide follow-up study from Sweden. Lancet 2012;379:244-9.

8. Chung WS, Lin CL, Sung FC, Hsu WH, Yang WT, Lu CC, et al. Systemic sclerosis increases the risks of deep vein thrombosis and pulmonary thromboembolism: a nationwide cohort study. Rheumatology 2014;53:1639-45.

9. Schoenfeld SR, Choi HK, Sayre EC, Avina-Zubieta JA. Risk of pulmonary embolism and deep venous thrombosis in systemic sclerosis: a general population-based study. Arthritis Care Res 2016;68:246-53.

10. Johnson SR, Granton JT, Tomlinson GA, Grosbein HA, Le T, Lee P, et al. Warfarin in systemic sclerosis-associated and idiopathic pulmonary arterial hypertension. A Bayesian approach to evaluating treatment for uncommon disease. J Rheumatol 2012;39:276-85.

11. van den Hoogen F, Khanna D, Fransen J, Johnson SR, Baron M, Tyndall A, et al. 2013 classification criteria for systemic sclerosis: an American College of Rheumatology/European League against Rheumatism collaborative initiative. Arthritis Rheum 2013; 65:2737-47.

12. Hoeper MM, Bogaard HJ, Condliffe R, Frantz R, Khanna D, Kurzyna M, et al. Definitions and diagnosis of pulmonary hypertension. J Am Coll Cardiol 2013;62 (25 Suppl):D42-50.

13. Soowamber ML, Granton JT, Bavaghar-Zaeimi F, Johnson SR. Online obituaries are a reliable and valid source of mortality data. J Clin Epidemiol 2016;79:167-8.

14. Hussein H, Lee P, Chau C, Johnson SR. The effect of male sex on survival in systemic sclerosis. J Rheumatol 2014;41:2193-200.

15. Pasarikovski CR, Granton JT, Roos AM, Sadeghi S, Kron AT, Thenganatt J, et al. Sex disparities in systemic sclerosis-associated pulmonary arterial hypertension: a cohort study. Arthritis Res Ther 2016;18:30.

16. Beckman MG, Hooper WC, Critchley SE, Ortel TL. Venous thromboembolism: a public health concern. Am J Prev Med 2010;38:S495-501.

17. Pattanaik D, Brown M, Postlethwaite AE. Vascular involvement in systemic sclerosis (scleroderma). J Inflamm Res 2011;4:105-25.

18. Dalleywater W, Powell HA, Fogarty AW, Hubbard RB, Navaratnam V. Venous thromboembolism in people with idiopathic pulmonary fibrosis: a population-based study. Eur Respir J 2014;44:1714-5.

19. Merashli M, Alves JD, Ames PR. Clinical relevance of antiphospholipid antibodies in systemic sclerosis: a systematic review and meta-analysis. Semin Arthritis Rheum 2017;46:615-24. 